\title{
LINEAMIENTOS BASICOS DE REASENTAMIENTO EN EL MUNICIPIO DE TUMACO COMO PARTE DEL PROCESO DE ORDENAMIENTO TERRITORIAL DEL NUEVO TUMACO
}

\author{
GABRIELA ISABEL MEJIA VALLEJO \\ Universidad Nacional de Colombia, Bogotá, Colombia. \\ Director: Doctor. Rene Carrasco Rey \\ E-mail: gaimejiava@unal.edu.co, zgaby33@gmail.com
}

\section{Resumen}

El riesgo en que están cerca de100 mil personas en la costa Pacífica, especialmente en los municipios de Tumaco, Nariño y Buenaventura, Valle del Cauca, hace necesario que el gobierno nacional diseñe una política pública para reubicar a esta población en zonas seguras y legales. No obstante, ese reasentamiento necesita la evaluación de múltiples factores sociales, económicos y ancestrales, así como una reestructuración de la coordinación entre las autoridades locales y nacionales para garantizar la recuperación del litoral y la protección del mismo.

La vulnerabilidad de la población provocó que se iniciaran los estudios que permitan superar este riesgo y la alcaldía de Tumaco junto con el gobierno nacional colombiano planteó un plan de reasentamiento, denominado Nuevo Tumaco que parte del Plan de Ordenamiento Territorial POT del municipio de Tumaco $2008-2019$, donde se contempla el desarrollo de tres fases de reasentamiento de sectores ubicados en zona de alto riesgo de la población en las tres islas de este municipio.

El presente trabajo busca desarrollar lineamientos de reasentamiento que contribuyan al proceso de ordenamiento territorial del Nuevo Tumaco, y aporten en la investigación de lineamientos generales de reasentamientos poblacionales provocados a causa de fenómenos naturales de Colombia.

Palabras claves: Reasentamiento urbano, Gestión del riesgo, Lineamientos de reasentamiento.

\section{ABSTRACT}

The risk of close to100 thousand people on the Pacific coast, especially in the municipalities of Tumaco, Nariño and Buenaventura, Valle del Cauca, makes it necessary for the national government to design a public policy to relocate this population in safe and legal areas. However, such resettlement requires the assessment of multiple social, economic and ancestral factors, as well as a restructuring of coordination between local and national authorities to ensure recovery and protection of the coast.

The population's vulnerability led to the start of studies to overcome this risk and the mayor of Tumaco together with the Colombian national government proposed a resettlement plan, called Nuevo Tumaco that starts from the POT Territorial Planning Plan of the municipality of Tumaco 2008 - 2019, which contemplates the development of three phases of resettlement of sectors located in high risk area of the population in the three islands of this municipality.

The present work seeks to develop resettlement guidelines that contribute to the territorial planning process of the Nuevo Tumaco and contribute in the investigation of general guidelines for population resettlement caused by natural phenomena in Colombia.

Keywords: Urban resettlement, Risk management, Resettlement guidelines. 


\section{Gestión de riesgos en Colombia}

Dada la localización y características geográficas del país, Colombia enfrenta amenazas por las condiciones geológicas, geomorfológicas e hidrometeorológicas.

El país está ubicado en una zona de alta complejidad geológica como producto de los movimientos de tres placas tectónicas que generan actividad sísmica y volcánica, con trece volcanes activos. Estas características explican la ocurrencia de fenómenos como los sismos y tsunamis, hechos registrados desde el año 1557 a 2008 (Contraloría General de la Nación, 2005: 209, citado por Poveda, 2011), con un balance de nueve terremotos de gran magnitud ocurridos en las zonas Pacífica y Andina, dos tsunamis en el sur de la costa Pacífica. Los sismos constituyen la principal amenaza natural debido a que cerca del $86 \%$ de la población está ubicada en zonas de nivel medio y alto de amenaza sísmica (DNP, 2001: 4, citado por Poveda, 2011).

Las inundaciones son los eventos que se presentan con mayor frecuencia, pero los terremotos son los que más causan pérdidas de vidas humanas y económicas. Estos tipos de desastres afectan de manera diferenciada las regiones geográficas. Por ejemplo, durante el periodo estudiado en la zona Andina ocurrieron la mayoría de deslizamientos (83\%) y el mayor número de incendios forestales $(99,26 \%)$. Por su parte en las regiones Caribe y Pacífica prevalecen los eventos de inundaciones (43\%).

Las consecuencias de estos desastres, así como los costos de la atención de la emergencia y la reconstrucción, obligaron al país a revisar los modelos de atención y el marco legal e institucional con el fin de desarrollar un sistema de prevención de desastres, además de programas de emergencia y rehabilitación (Poveda,2011,p.125).

\begin{tabular}{|c|c|c|c|c|c|c|}
\hline \multicolumn{2}{|c|}{$\begin{array}{l}\text { IDENTIFICACIÓN } \\
\text { DEL EVENTO }\end{array}$} & \multicolumn{5}{|c|}{ AFECTACIÓN DEL EVENTO } \\
\hline Evento & Año & $\begin{array}{c}\text { No de } \\
\text { Municipios } \\
\text { afectados }\end{array}$ & Área Km3 & Muertos & Heridos & $\begin{array}{c}\text { No. } \\
\text { Personas } \\
\text { Afectadas }\end{array}$ \\
\hline $\begin{array}{l}\text { Maremoto y } \\
\text { Tsunami en } \\
\text { Tumaco }\end{array}$ & 1906 & 12 & N.R. & $500-1500$ & N.R. & N.R. \\
\hline $\begin{array}{c}\text { Maremoto y } \\
\text { Tsunami en } \\
\text { Tumaco }\end{array}$ & 1979 & 7 & N.R. & 452 & 1011 & 7.476 \\
\hline $\begin{array}{l}\text { Sismo Atrato } \\
\text { Medio }\end{array}$ & 1992 & 33 & $18.708-39.396$ & 26 & 80 & 28.500 \\
\hline
\end{tabular}

Tabla 1. Eventos Naturales en Colombia Elaboración propia a partir de los datos del INE

\section{EI reasentamiento de población: una estrategia para la prevención y reducción del riesgo}

Para esta investigación, al hablar de re-asentamiento, se adoptara el concepto de Anne Catherine Chardon. Esta autora plantea "reflexionar sobre el significado de los verbos "asentar" y "asentarse" en el contexto humano, los cuales por supuesto hacen referencia al hecho de establecer o establecerse en un sitio, pero con una connotación particular, esto es la seguridad, la firmeza, la permanencia y durabilidad en el tiempo. Esta permanencia lleva a comentar que el hecho de asentarse no es repentino sino que resulta de un proceso tanto espacial o físico-espacial como social, cultural, económico, puesto que corresponde a la fundación de un lugar, de una comunidad, de un asentamiento y finalmente de un hábitat con sentido de arraigo" (Chardon,2010)

El concepto de reasentamiento es sobre todo objeto de reflexión en instituciones internacionales, como el Banco Mundial y el Banco Interamericano de Desarrollo que han participado de la financiación de numerosos proyectos de desalojo-desplazamiento, quienes asumen el reasentamiento como una estrategia de reducción del riego, y establecen criterios para el diseño y evaluación del plan de reasentamiento, tales como Información base de la población, participación de la comunidad, medio ambiente, seguimiento y evaluación, entre otras, todas de carácter técnico, pero también para la financiación de los proyectos se exige cada vez que los procesos no se limiten a los aspectos físicos, sino que también, "abarquen dimensiones sociales, culturales y económicas, entre otras, en pro del desarrollo sostenido en el tiempo de la comunidad afectada", menciona Chardon. 
Entre las diferentes estrategias de prevención de riesgo que se adelantan en el país se encuentra el reasentamiento de poblaciones localizadas en áreas de riesgo no mitigable, es decir, aquellos riesgos que no pueden reducirse con ninguna otra medida o que su costo resulta tan alto que es más económico reasentar a la población.

Entre los reasentamientos preventivos que se han llevado a cabo se destacan los del municipio San Cayetano, en Cundinamarca, con 10.000 personas, en la ciudad de Medellín, de 45.000 personas, y Bogotá, de 65.000 personas, aproximadamente.

Un estudio sobre las experiencias y prácticas de reasentamiento elaborado por el Departamento Nacional de Planeación (DNP) en 2005 (DNP, ACCl, 2005, citado por Poveda, 2011) mostró que entre los años 1994 y 2004 se desplazaron aproximadamente 130.00 personas (28.555 hogares), en 192 municipios, por desastres naturales.

Este estudio también encontró en una muestra realizada en 137 municipios (9,6\% del total del país), que tenían previstos 158 planes de re sentamiento para 95.340 familias, que habitaban en zonas de riesgo, los cuales se debían ejecutar hasta el año $2011,81 \%$ de ellas en la región Andina, $13 \%$ en la pacífica y $6 \%$ en las otras regiones. Infortunadamente no existen datos consolidados de los avances en la ejecución de estos planes de reasentamiento, ni un sistema de información nacional sobre el tema, sino solamente datos dispersos en cada uno de los municipios (Poveda, 2011, p.127).

\section{Gestión de riesgos en Tumaco}

Tumaco pertenece a la subregión pacifica sur, cerca del litoral de la subducción del Pacifico, donde colisionan las placas tectónicas de Nazca y Suramericana dentro del cinturón de Fuego del Pacifico, siendo esta la parte de zona sísmica más activa del planeta.

La presencia de las placas en la costa Pacífica, ha provocado cinco tsunamis, el primero ocurrió el 31 de Enero de 1906 (magnitud 8,8 en la escala de Richter), el 2 de Octubre de 1933 se produjo otro sismo (magnitud 6,9 en la escala de Richter), luego se presentaron otros dos, el 14 de Mayo de 1942 (magnitud 7,9 en la escala de Richter) y el 19 de Enero de 1958 (magnitud 7,8 en la escala de Richter); el ultimo Tsunami ocurrió el 12 de Diciembre de 1979 (magnitud 7,7 en la escala de Richter).

Según el informe de la Dimar de 2013 El terremoto y tsunami ocurrido en el año 1906 registro una afectación de 500 a 1500 muertos, el Tsunami de 1979, registra 452 muertos, 1011 heridos, 1080 viviendas destruidas en Tumaco y 700 viviendas averiadas. La población de Tumaco se encuentra en un grado significativo de vulnerabilidad y exposición frente a un fenómeno de amenaza natural como es el tsunami.

\section{El reasentamiento de población en Tumaco}

Por motivo de riesgo de amenaza alta de Tsunami e Inundación. EL Plan de Ordenamiento Territorial del municipio de Tumaco 2008 - 2019 ha contemplado el desarrollo de tres Fases de Relocalización de sectores ubicados en zona de alto riesgo de la población de las tres islas del municipio de Tumaco. El territorio para la relocalización está comprendido entre el puente de Agua Clara ( $\mathrm{Km} \mathrm{16})$ hasta el Km 92, en donde limita con el municipio de Barbacoas en la vía panamericana Tumaco - Pasto. Sobre la margen izquierda de este recorrido limita con la ensenada y el rio Caunapi, mientras que por la margen derecha con el rio Guiza y el rio Mira.

La zona de relocalización de la primera fase de reasentamiento de población, el Nuevo Tumaco, es el corregimiento de Chilvi, conformado por las comunidades, Agua Clara, Bucheli y Bajo Chilvi. 


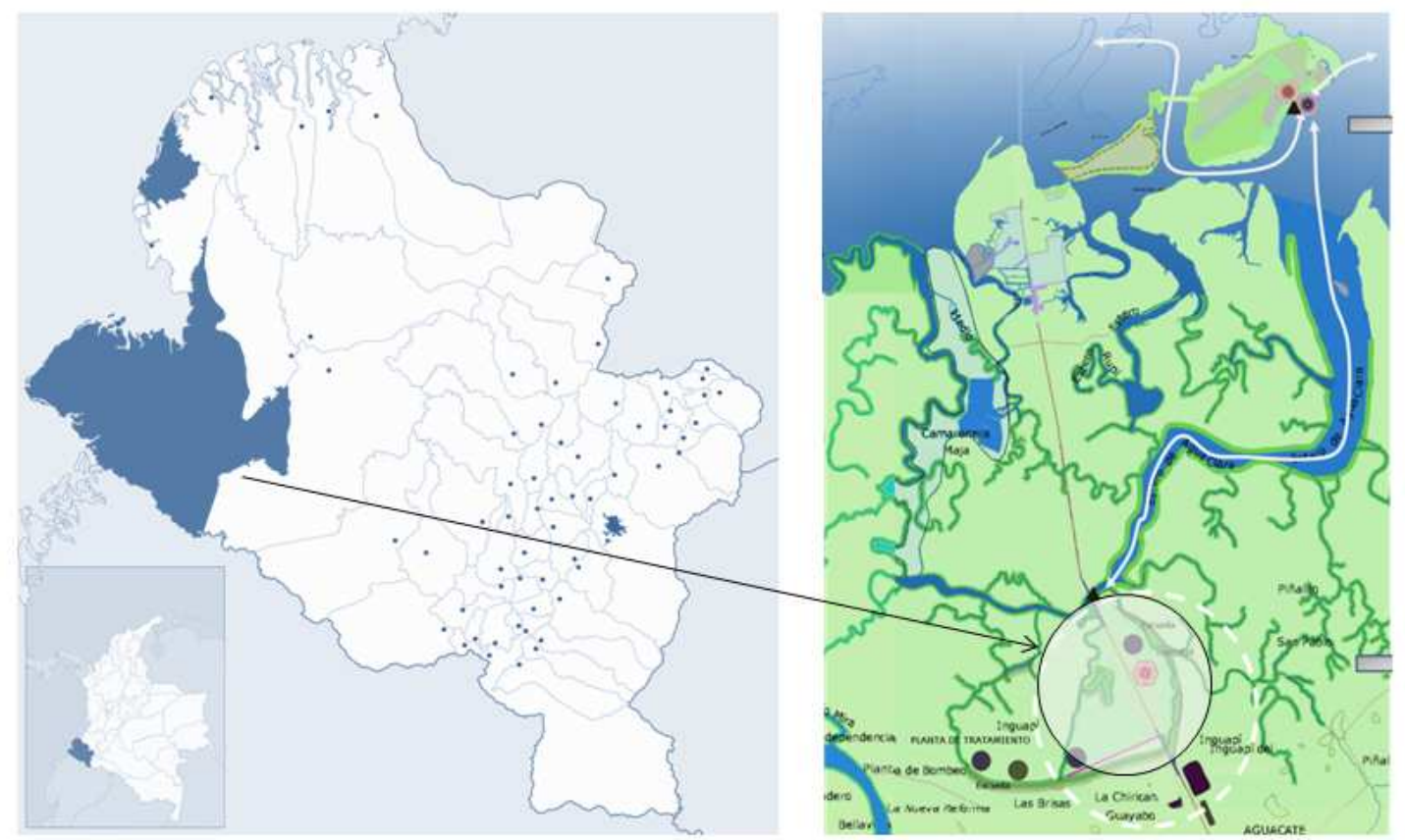

Figura 1. Plano de Localización del Nuevo Tumaco en el Municipio de Tumaco

Fuente: Elaboración Propia a partir de la información del municipio de Tumaco POT 2008 - 2019

El área de Chilvi corresponde a una zona de desarrollo, como el plan la ha catalogado, Unidad De Actuación Urbanística de la Primera Fase de Relocalización. Se define como la zona prioritaria para el desarrollo de proyectos urbanísticos de relocalización de las viviendas que se encuentran en alto riesgo. Esta unidad se encuentra en la Zona del Nuevo Tumaco, 380 has, identificada en el siguiente mapa.

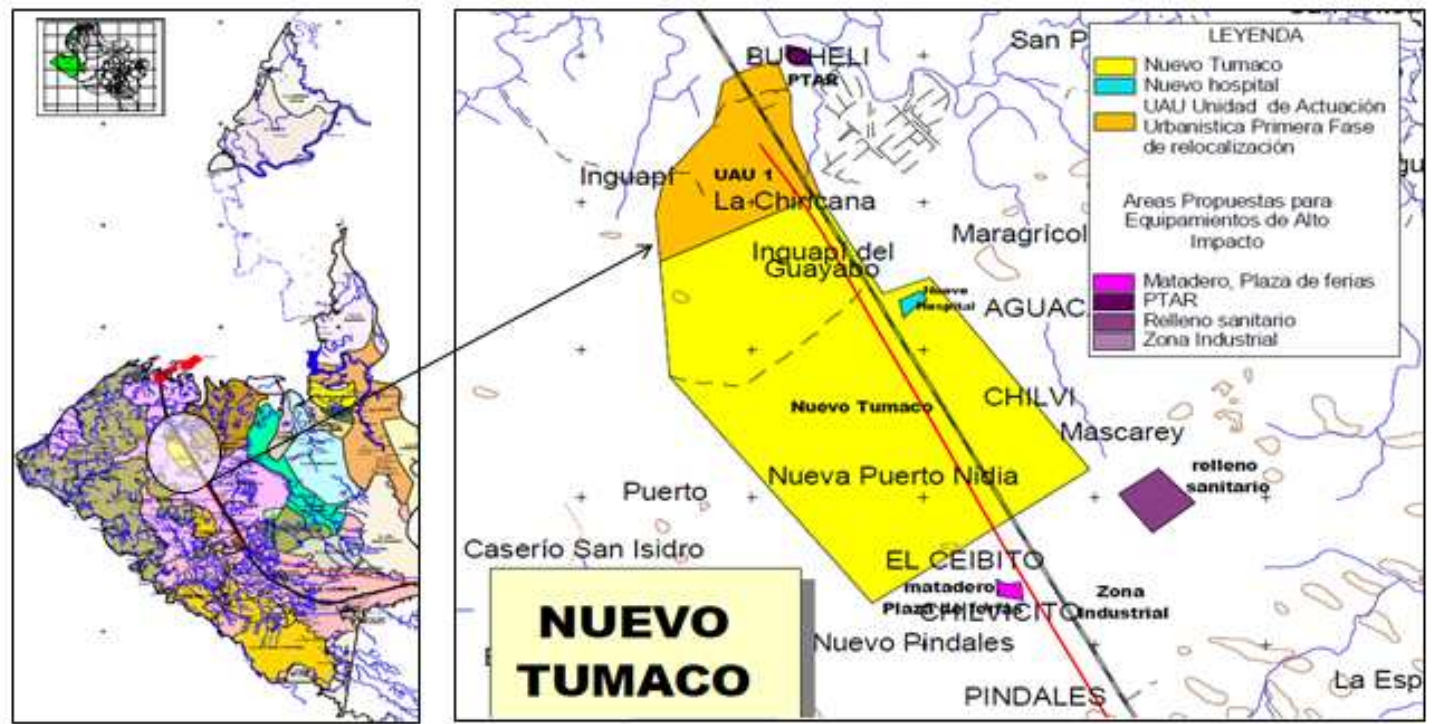

Figura 2. Plano de Localización del Nuevo Tumaco- Reasentamiento Poblacional de las Islas de Tumaco

Fuente: Tomado del El Plan de Ordenamiento Territorial del municipio de Tumaco 2008 - 2019

En el corregimiento de Chilvi existen diferentes medios de transportes para la accesibilidad, equipamientos como el hospital y la escuela y características socioeconómico, físico-biótico, técnicas- ambientales, así como tipo de suelo, (terrazas Fluviodeltáicas), similares a la de las islas de Tumaco. Asociación climática cálida húmeda con topografía plana, hace de este sector, un terreno apto para el desarrollo urbano, además posee fácil suministro de agua corriente a través de un acueducto y facilidad para el desarrollo de sistemas de tanque séptico con filtros anaeróbicos para el manejo de aguas negras 


\section{Referentes de Reasentamiento}

Se han conocido procesos de reasentamiento muy destacados en el mundo por razones de amenaza natural para nuestro estudio tomaremos aquellos casos relevantes de riesgo por tsunami que se reasentaron en sitios más seguros y que dicho proceso fue orientado por algunos lineamientos que se constituyen en un referente a tener en cuenta para considerarlos en nuestro contexto costero de Tumaco.

\section{Referentes de Reasentamiento en Japón}

Minamisanriku es un pueblo y puerto de la prefectura de Miyagi, Japón. Situado en la costa este del país, cubre un área de 163,74 km² y su población es de 17.393 habitantes a 1 de enero de 2011.

Minamisanriku ha sido golpeado por tsunamis de alta magnitud durante los siglos pasados, incluyendo uno importante en 1896, 1933, y 1960. La ciudad fue duramente golpeada por el tsunami que siguió al terremoto de marzo de 2011. Según las cifras del Gobierno japonés, en Minamisanriku fallecieron cerca de 1275 personas, y hubo cerca de 278 desaparecidos, aunque en este caso la cifra es variable.

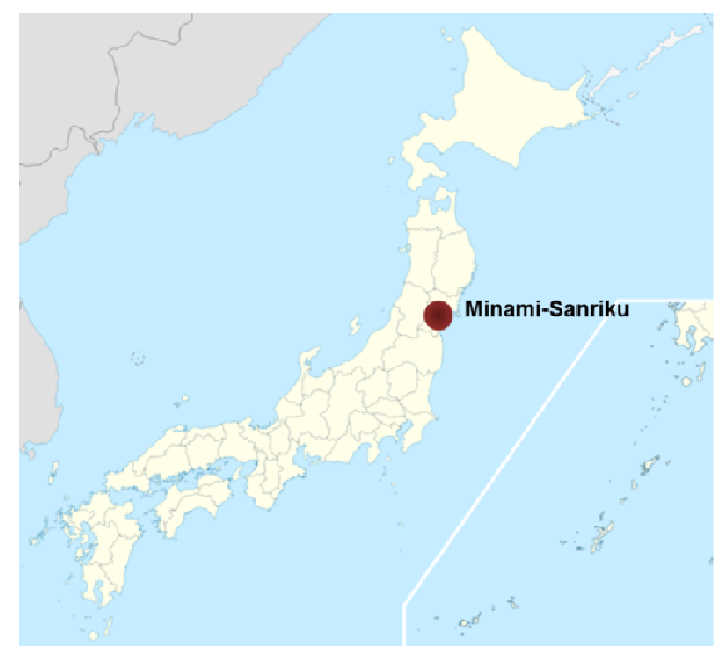

Figura 3. Localización Minami Sanriku

Fuente: Elaboración propia

Debido a las diversas experiencias que tiene Japón en materia de gestión del riesgo y reasentamiento a causa de la gran cantidad de tsunamis y terremotos se incluye dentro de la investigación el caso de estudio de población de Minamisanriku que es reasentada en el año de 1933 en el noreste de Japón.

La figura 4 ilustra la transición de la propiedad de vivienda después del tsunami de 1933 en el distrito de Hyogo, Iwate Prefectura (Murao y Isoyama, 2012,). Debido a la estrategia de reubicación, casi no hay construcción en 1948, excepto en el lugar más alto proporcionado por el gobierno. Sin embargo hay que reconocer que muchos edificios se han construido poco a poco en las tierras bajas vulnerables en el siglo veinte (Murao, 2012).

El tsunami de 2011 atacó el distrito y se llevó cientos de nuevas construcciones de las tierras en la parte baja como se muestra en la figura 6 . Por otro lado, las casas en el reasentamiento superior, realizado por el gobierno después del tsunami de 1933 sobrevivió al tsunami destructivo (Murao, 2012). 

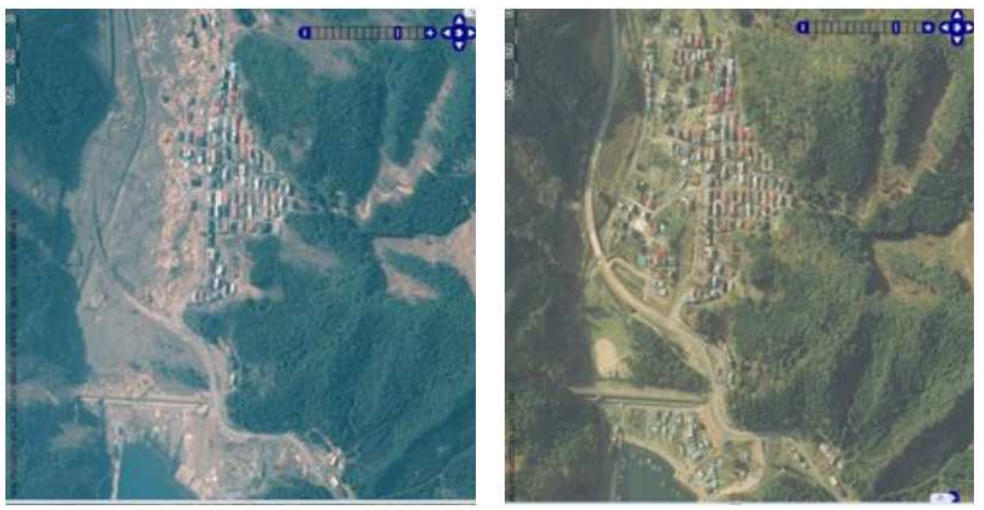

Figura 4. La situación de daño edificio en el Congo por el tsunami de 2011 (izquierda) con pre-tsunami Fuente: Murao, 2012

El hecho de que el reasentamiento en la planta más alta proporcionada por la planificación de recuperación tras el tsunami y la política después del tsunami de 1933 no fue dañado por el tsunami de 2011 pone de manifiesto la importancia del uso de la tierra de mitigación para la reducción de desastres del tsunami. Esta exitosa experiencia en la zona costera propensa a los tsunamis debe trascender hacia el futuro (Murao, 2012).

Todos los distritos dañados idearon su plan de recuperación que permite reducir el riesgo de futuro tsunami basado en la experiencia pasada en Japón. Especialmente la participación pública ha sido reconocida como un factor importante para hacer un plan de recuperación desde el gran terremoto de Kobe de 1995.

Cada municipio hizo dos tipos de simulación de tsunami dependiendo de la posibilidad de ocurrencia para hacer planes de reasentamiento. El gobierno japonés prepara 12 proyectos que se muestran en la Fig.5 para apoyar la realización de los planes. (Murao, 2011).

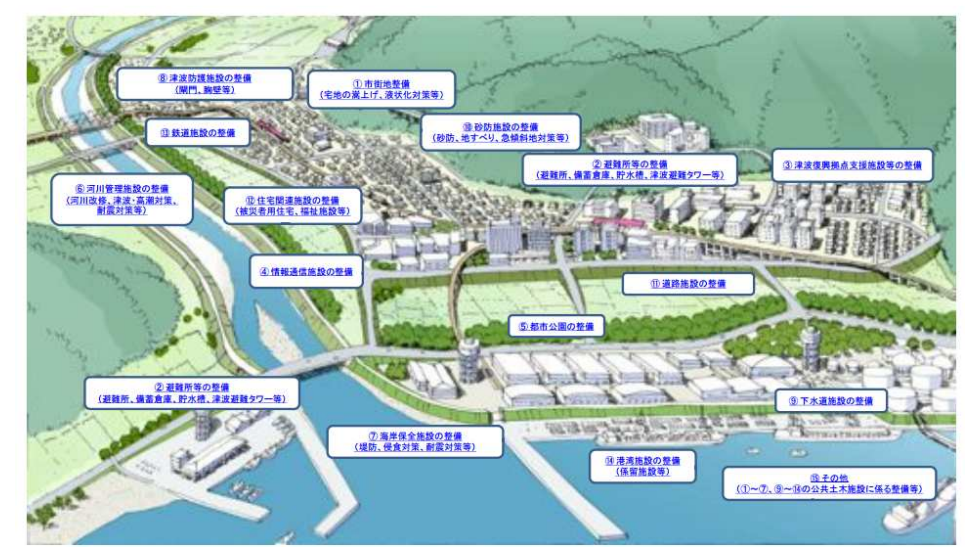

Figura 5. Proyectos de Recuperación después de Tsunami.

Fuente: Murao, 2012

Algunos de las recomendaciones que Murao hace para futuros reasentamientos se presentan a continuación:

1. Traslado a tierras más altas del área de la ribera como una estrategia de recuperación después del tsunami debe llevarse a cabo con adquisición de tierras por la compra de los gobiernos nacionales y locales para evitar futuras uso privado del espacio vulnerable frente al mar.

2. Debe estimarse la BCP Business Continuity Planning, también denominada empresa de continuidad y planificación flexible como red que es indispensable para mantener la cadena de suministro.

3. El Refuerzo de edificios es esencial para reducir el riesgo de colapso. Promoción, educación y objetivos cuantitativos de medio ambiente son importantes. 
4. Planificación de recuperación ante los desastres es un proceso importante para llegar a cabo un acuerdo para tener una visión de futuro del distrito entre el gobierno local y los residentes. Compartir la visión de futuro antes de un desastre puede evitar un conflicto emocional en la etapa de realización y el plan de recuperación post-desastre.

5. Estimación de recursos disponibles, así como estimación de daños de desastres por adelantado es necesario llevar a cabo actividades de reconstrucción. Debe ser considerado por el nivel nacional, local y comunitario según el nivel de daño.

\section{Estrategias de Reasentamiento en la Región de Biobio - Chile}

\section{Región del Biobío}

Es una de las quince regiones en las que se encuentra dividido política-administrativamente Chile. Limita al norte con Región del Maule, al este Argentina, al sur Región de la Araucanía y al oeste el océano Pacífico.

Cuenta con una superficie de $37068,7 \mathrm{~km}$ y una población de 2114286 habitantes según la proyección del INE de 2015, siendo la segunda región más habitada del país. La región está compuesta por las provincias de Arauco, Biobío, Concepción y Ñuble. Su capital regional y ciudad más poblada es Concepción. El terremoto y tsunami de Chile en 2010 dejó 126 víctimas y 25 desaparecidos, tuvo una magnitud de 8,8 grados, una duración de 3 minutos y olas de hasta 30 metros.

\section{Plan de Reconstrucción Urbana del Borde Costero}

La reconstrucción del borde costero surge como una necesidad inminente ante la devastación provocada por el terremoto del 27 de febrero de 2010. Después de haber organizado como voluntario el plan para revisar y calificar el estado de las edificaciones de la ciudad de Concepción, se abrió para Baeriswyl un frente insospechado y un desafío profesional mayor, causado por la destrucción urbana provocada por el maremoto en 18 localidades costeras de la región del Biobío, entre ellas ciudades tan consolidadas como Talcahuano y Dichato, y otras tan pequeñas como caletas pesqueras.

Por encargo de la Intendencia Regional del Biobío, se creó el Plan de Reconstrucción Urbana del Borde Costero (PRBC 18), cuya coordinación general es solicitada a Baeriswyl, quien conformó un pequeño equipo de trabajo estructurado en tres áreas de intervención territorial; Área Norte, Centro y Sur, dirigidos por Iván Cartes, Waldo Martínez y Carolina Arriagada respectivamente. Así el PRBC18 se constituyó en una organización descentralizada transformándose en el eje de coordinación para las decisiones e intervenciones de reconstrucción en el ámbito urbano y multisectorial.

La catástrofe del 27 de febrero provocó destrucción y pérdidas de incalculable magnitud para todo el país. El tsunami que acompañó a este sismo fue más allá y destruyó ciudades, borrando en algunos casos el tejido urbano, que en el pasado diera forma a un paisaje edificado. De las 36 localidades urbanas que conforman la constelación de asentamientos costeros de la región del Bío Bío, 18 experimentaron daños de gravedad, y en varios casos tardaron más de un año en restablecer sus condiciones básicas de funcionamiento; vale decir los sistemas de comunicación terrestre y transporte público, redes de servicios y equipamientos públicos. Pero en otros casos más extremos, la ciudad fue parcialmente devastada y no fue posible su recuperación sin mediar una estrategia de diseño que permita asegurar una mayor resiliencia de lo reconstruido.

El registro de daños de esta catástrofe da cuenta de 11.633 edificaciones destruidas, en su gran mayoría residenciales, pero también con destinos productivos, comerciales y de servicios. Los daños involucran más de 1.550 entidades comerciales, 570 productivas, 42 educacionales y cinco centros de salud. Como consecuencia de estos daños se vio seriamente comprometida la vida urbana de más de 240.000 habitantes que, según el censo de 2002, residían en estas ciudades costeras.

Mientras las ciudades mediterráneas vieron el avance de la reconstrucción a través de la puesta en marcha de los planes y programas de reconstrucción de viviendas e infraestructura, en las localidades costeras esto no fue posible, ya que previamente fue necesario redefinir los roles de las localidades afectadas, los usos de suelo, los emplazamientos seguros para la infraestructura crítica y en algunos casos reurbanizar, es decir, un plan para reconstruir ciudades como un todo. 


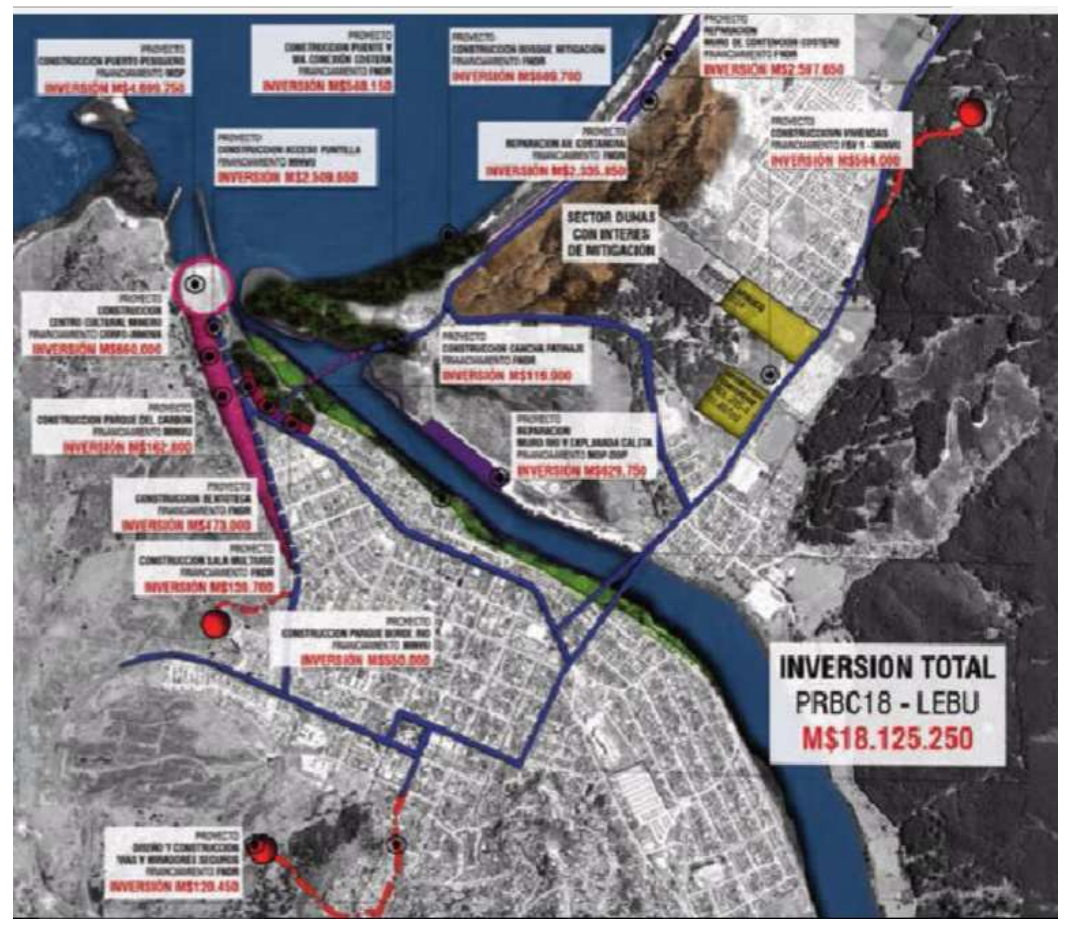

Figura 6. Proyectos de Recuperación Bio-Bio

Fuente: PRBC 18

El Plan de Reconstrucción del Borde Costero del Litoral Biobío (PRBC 18), planteó organizar la reconstrucción como un proceso urbano de calidad, inclusivo e integral. Entre los objetivos del PRBC 18 se destacaron:

- Una reconstrucción correctiva y de calidad, que permitiera incorporar agentes de valorización urbana donde antes no existía.

- Una reconstrucción inclusiva, que reflejara de manera autentica los anhelos de sus residentes.

- Una reconstrucción que promoviera el máximo estándar de seguridad posible, que permitiera la recuperación del hábitat urbano costero, mejorando la resiliencia urbana.

- Una reconstrucción que fortaleciera la identidad y que reconociera los valores locales.

- Una reconstrucción que en lo posible entregara oportunidades de las más diversas actividades económicas para sus habitantes y creara oportunidades de futuro (Baeriswyl, S. 2013 citado por Cartes. 2014)

La convergencia de estos objetivos se dio en el diseño de 18 planes maestros, para los cuales se determinó la estrategia de reconstrucción urbana, las medidas de mitigación, las zonas seguras, el emplazamiento de equipamientos críticos, las vías de evacuación, las tipologías residenciales más resilientes y los proyectos detonantes para la recuperación productiva (Cartes. 2014).

Estas recomendaciones se entregaron por Dr. Sergio Baeriswyl en su participación en el marco del concurso convivE VII en la ciudad de Bogotá en el 2013.

1. Difícilmente una ciudad en el planeta pueda ser invulnerable a los efectos devastadores de catástrofes naturales o cambios inesperados en los ecosistemas.

2. El crecimiento urbano ejerce una enorme presión sobre los ecosistemas y deja a las ciudades en una condición de mayor vulnerabilidad.

3. La catástrofe en Chile del año 2010 obligo a los planificadores a replantearse las metodologías tradicionales aplicadas a la concepción y diseño de ciudades.

4. Para el reasentamiento y nueva planificación realizada en Chile es indispensable asumir altos costos sociales, políticos y económicos. 
5. Resiliencia urbana significa mejorar la capacidad de una estructura urbana para recuperarse luego de una perturbación, como en este caso un terremoto y tsunami.

6. El cambio más sensible en la estructura tradicional de ciudad, es aquel que valora la vista y el contacto directo con el paisaje y se niega a renunciar a este derecho.

7. Los costos de la mitigación desbordaron la ecuación de rentabilidad, y se pensó en el reasentamiento de las familias afectadas.

8. Para Chile se plantearon Planes Maestros y de Mitigación para atenuar los efectos destructivos sobre las ciudades y mejorar su resiliencia. haciendo verificación hidrodinámica de las estructuras urbanas.

9. Algunos criterios para el nuevo ordenamiento territorial Chileno fueron: Se incorporó por primera vez al riesgo de tsunami en los Planes reguladores (Planes parciales) para la relocalización de infraestructura crítica. Reconocimiento de la vulnerabilidad del territorio en los instrumentos de planificación territorial y finalmente culturizar a los residentes, estructuras productoras locales y composición del tejido social.

\section{Propuesta de Lineamiento básicos}

\section{Reasentamiento, Hábitat e imagen de la Ciudad}

El que hacer del arquitecto se fundamenta desde los principio de Heidegger en aprender a habitar, como lo explica Saldarriaga "Vivir es habitar y habitar es vivir. La experiencia de la arquitectura comienza y termina en todos los actos que contribuyen el habitar [...] vivir es una constante interacción entre el ser humano y el mundo en la que la arquitectura asume un papel especial. La experiencia de la arquitectura no es accidental o periférica, se encuentra en el centro mismo del acto de vivir" (2002, pág. 181). El oficio del arquitecto, urbanista y el mío propio en el campo de investigación de la ciudad, el urbanismo y la arquitectura será el contribuir en el proceso de habitar del hombre.

Algunos autores como Hurtado explican la integralidad que existe en el concepto de hábitat, entendiendo este como el espacio creado para que el hombre pueda habitar cómodamente. Tarchópulos y Ceballos [2003] cuando expresan que "La habitabilidad es, en pocas palabras, la condición satisfactoria de calidad ambiental, material y cultural del espacio habitado por las personas. Es un concepto que se ha definido basándose en dos tipos de criterios. Uno derivado de los parámetros acumulados a lo largo del proceso modernizador y el otro proveniente del medio cultural específico donde se habita. El primero se ha traducido en normas o estándares generalizados referentes a aspectos cuantitativos del entorno: saneamiento básico, servicios públicos, accesibilidad, relación entre espacio libre y espacio construido, densidad habitacional, tamaño, estabilidad y eficacia de las unidades de vivienda. El segundo se relaciona con la valoración del entorno por parte de los habitantes y con su satisfacción en términos de seguridad, identidad, comodidad y apropiación".

Pero que sucede cuando una ciudad con su memoria e imagen debe ser reasentada, Saldarriaga al referirse a la imagen de la ciudad nos explica que, "la imagen no es fija o estática se ha transformado a lo largo de la historia y se transforma en el lapso de vida de una persona. Posee una dimensión espacio temporal que incorpora lo pasado, lo presente y lo posible. En todo ello la memoria desempeña un papel esencial. La memoria es en cierta forma, la imagen del mundo" (2002, pág. 162). El proceso de reasentamiento del Nuevo Tumaco se basa en el concepto de transformación de la imagen de la ciudad, a través de un proceso participativo que ayude a reducir el impacto del cambio de hábitat, en el nuevo hábitat en Chilvi fuera de la isla, se debe reducir todos los riesgos de que el nuevo hábitat perjudique o incomode a sus habitantes.

Para crear la imagen de la ciudad, existen una serie de factores y dentro de éstas se entienden tres componentes: las físicas, las sociales, urbanísticas y las ambientales. Que a su vez pueden ser resumidas en una sola frase mejor hábitat en un sitio seguro para su cultura. 


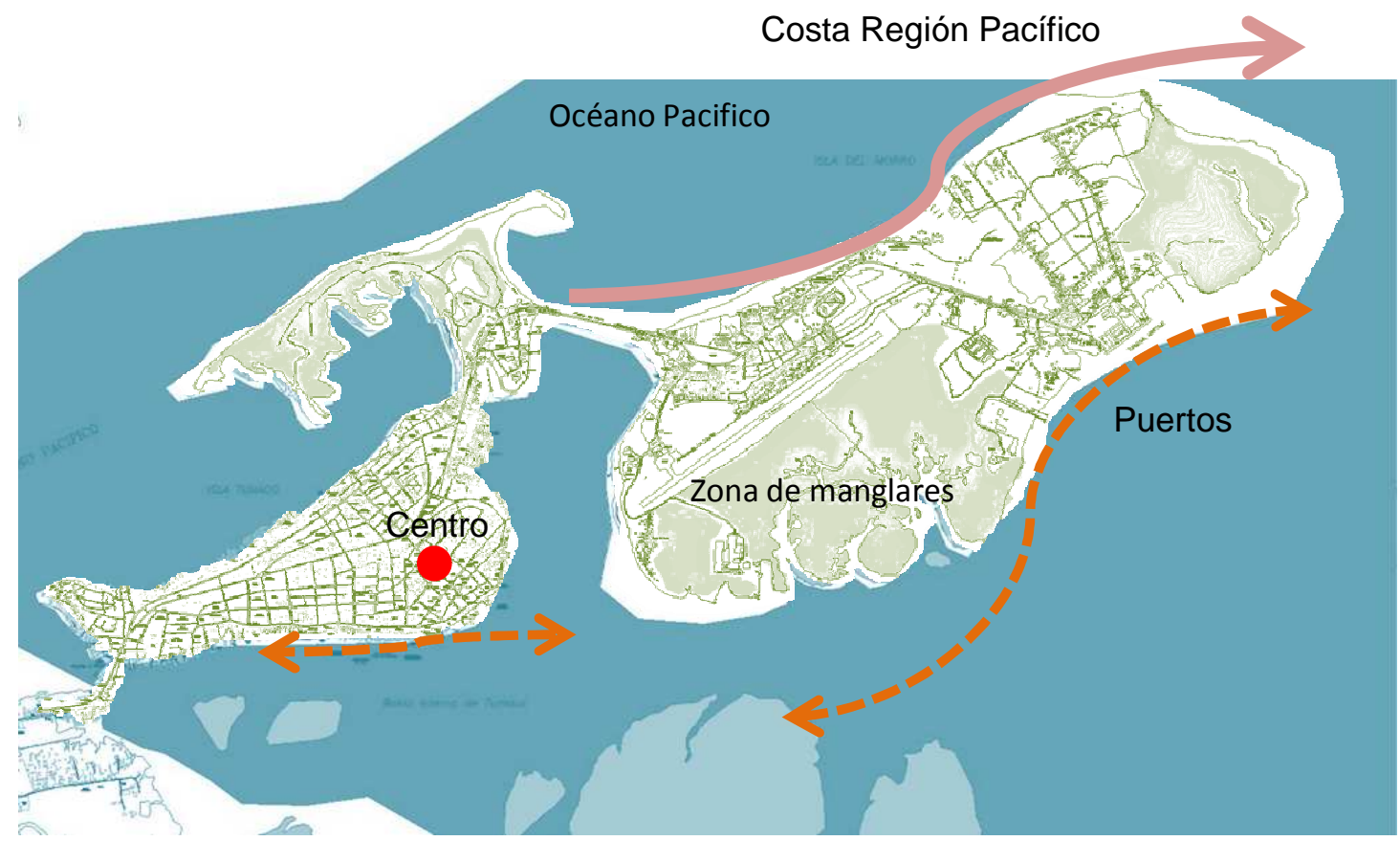

Figura 7. Proyectos para las Islas de Tumaco después del Reasentamiento

Fuente: Elaboración propia a partir de información de la Alcaldía de Tumaco

\section{Dimensiones de reasentamiento}

Racionalizando tanto la experiencia de Tumaco y los casos de estudios de reasentamiento de otros países proponemos unos lineamientos básicos para el reasentamiento de poblaciones en situación de riesgo, pero de manera particular para el municipio de Tumaco, para ello consideraremos unas tres áreas a tener en cuenta, desarrolladas de manera más específicas en dimensiones que aborde todos los aspectos (Figura No. 8).

Estas tres áreas básicas corresponde a la Físico ambiental, socio cultural y la Urbanística, áreas que nos permite agrupar los aspectos necesarios a considerar en los procesos de reasentamiento de la población, este modelo de clasificación nos orienta didácticamente nuestros trabajo de investigación.

Igualmente se propone cuatro dimensiones vitales; que se muestran en la figura No. 9. Estas dimensiones son, Dimensión Cultural relacionada a la Dimensión Socio económica porque la actividad que realizan cultural y ancestralmente es la pesca, y a su vez esta es una actividad económica y productiva; la Dimensión Urbanística está relacionada a la Dimensión físico ambiental, por cuanto se busca el desarrollo urbano sostenible en el Nuevo Tumaco. 


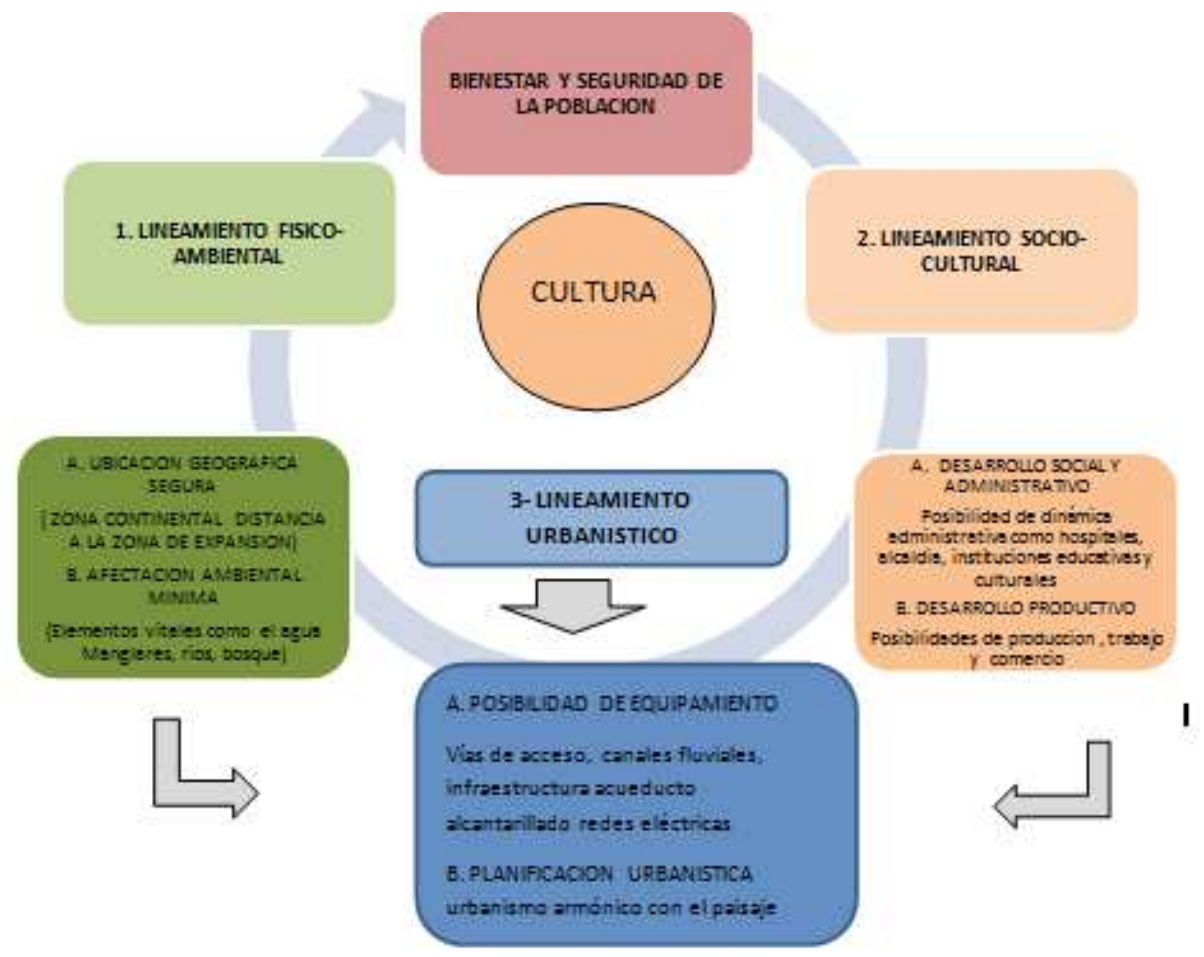

Figura 8. Lineamientos de reasentamiento Nuevo Tumaco. Fuente: Elaboración propia

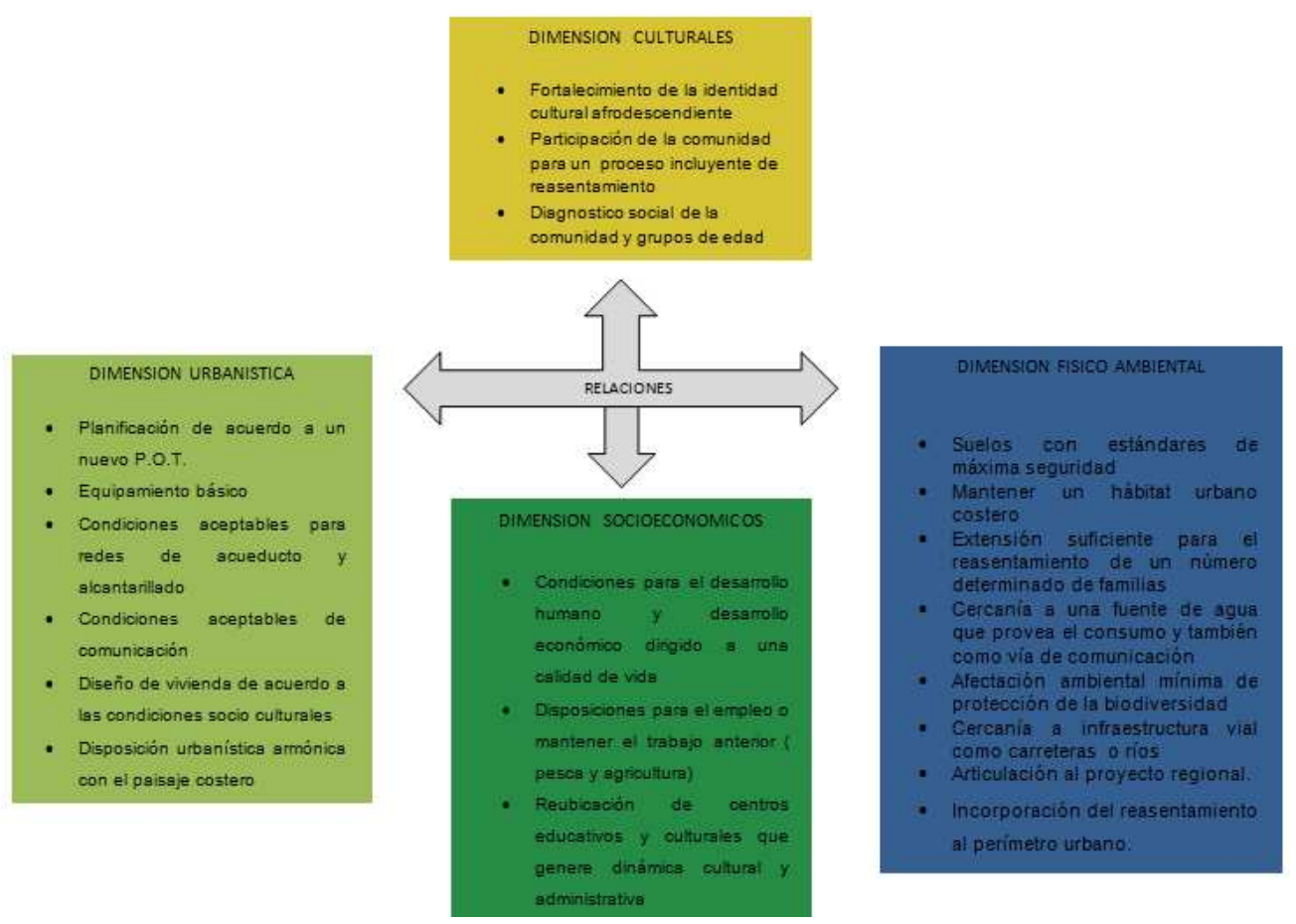

Figura 9. Dimensiones de reasentamiento Nuevo Tumaco. Fuente: Elaboración propia 


\section{Gestión social del Riesgo}

"La imagen del mundo para la humanidad es la imagen de un mundo hecho para habitar. Ese mundo es su hogar, en el que se encuentra lo necesario para la conservación de la vida y también aquello que atenta contra ella. La conciencia de la vida y de la muerte dan un sentido especial a esa imagen. [...]" (Sandarriaga, 2002).

El proceso de reasentamiento solo inicia cuando se establece el riesgo (para las personas) y la vulnerabilidad (sobre el territorio) por un fenómeno natural en particular. Saldarriaga menciona que "La estabilidad de la estabilidad de la imagen del mundo se fractura con aquello que dice el conocimiento. La fragilidad de la condición humana en el planeta, la fragilidad misma de la tierra en constante riesgo, [...]" (Sandarriaga, 2002, pág. 181).

Cuanto antes se inicie con el proceso de sensibilización, diagnóstico y participación para la formulación de los proyecto de reasentamiento más efectivo será el plan. Después que se formule el plan de reasentamiento se puede llevar a cabo la socialización de los proyectos y de esta manera se puede iniciar con un proceso de transformación de la imagen de la ciudad en el imaginario de la población a ser reasentada.

El plan de reasentamiento debe incluir al mayor número de actores posibles, se debe incluir un plan para los comerciantes que permita reestablecer las actividades comerciales de manera rápida y oportuna después de un evento natural sísmico. La gestión social del riesgo se basa en dignificar al ser humano cuando pase por la ocurrencia de un evento natural inesperado. El plan de reasentamiento debe respetar los derechos humanos fundamentales. La Cepal informa que "Durante los últimos treinta años, los huracanes, los terremotos, los deslizamientos y las inundaciones han causado pérdidas físicas en América Latina y el Caribe que alcanzan los US\$ 3,2 mil millones. En promedio, los desastres en la región han causado la muerte de más de 5.000 personas y afectado a 4 millones de personas cada año. (Cardona 2005)"

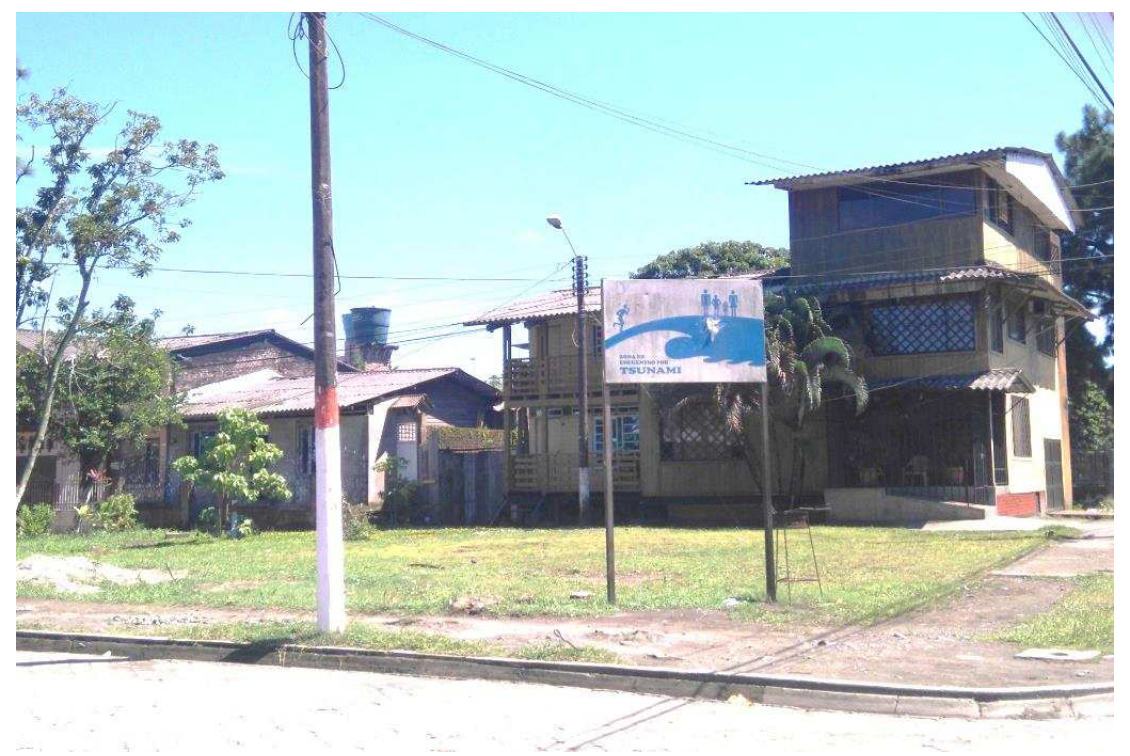

Figura 10. Foto de Plan de evacuación de Tsunami en el barrio la Ciudadela en el municipio de Tumaco Fuente: Elaboración propia

\section{Vivienda del Pacifico Colombiano en Tumaco}

Para la construcción de la vivienda en Tumaco se necesita aprovechar los atributos del territorio (accesibilidad fluvial) para suplir las necesidades de reasentamiento, esto equivale a pensar las distintas alternativas de la vivienda: materiales, sistema de agrupación y localización de la misma; también por el tipo 
de cultura del pacifico es posible capacitar a los habitantes del pacifico para que participen en la construcción de las viviendas debido a que es común que ellos realicen el proceso de construcción de su vivienda propia (autoconstrucción de vivienda).

Gilma Mosquera propone para mejorar la vivienda del pacifico colombiano: "el mejoramiento de los equipamientos y servicios colectivos existentes, la optimización de las relaciones entre el espacio público y el espacio privado, la superación de las deficiencias de saneamiento ambiental y provisión de agua para el consumo doméstico. Urbanísticamente su valor radica en que las formas de agrupación de viviendas nuevas siguen la lógica de organización espacial de las aldeas del pacifico" (Mosquera, 2011, pág. 180).

Con el traslado o reasentamiento de la población a un nuevo territorio, se debe conocer y estudiar los atributos del nuevo territorio, como equipamientos y servicios disponibles, la mayoría de veces el tipo de suelo cambia y es necesario explicar a los población reasentada de las actividades productivas que se pueden desarrollar en el nuevo territorio, sin embargo es importante que el territorio del reasentamiento sea similar al de la población que se está reasentando porque de esto depende gran parte de la adaptación social de la población.
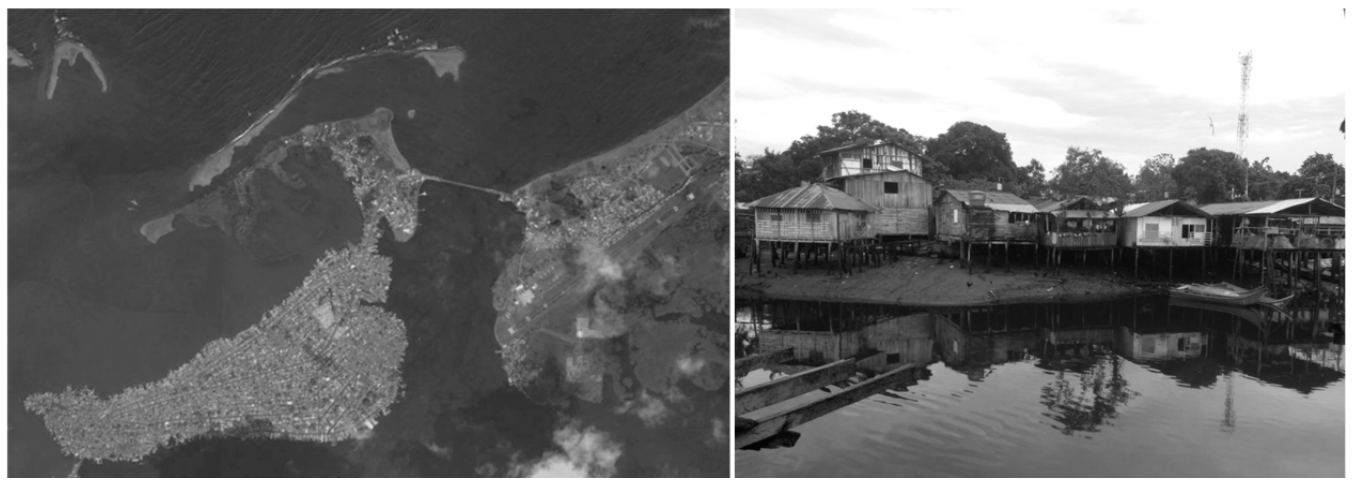

Figura 11. Foto aérea de la Isla de Tumaco y foto de viviendas palafiticas del municipio de Tumaco Fuente: Elaboración propia

\section{Fase de Planeación}

Consiste en el diagnóstico y evaluación del reasentamiento en cada una de las cuatro dimensiones que analiza el reasentamiento, descritas en la figura 9. El desarrollo de esta fase hace uso de un grupo de indicadores de tipo cualitativo y cuantitativo para los cuales es consultada la información de fuentes oficiales como la Procuraduría General de la Nación en el año 2008 y el Departamento Administrativo Nacional de Estadística (DANE), entre otros. Los datos son validados por medio de consultas y encuestas directas de esta investigación y ratificada con información de autoridades locales.

El proceso que se describe en la figura 11 muestra parte del proceso de planificación del reasentamiento, como punto de partida, un diagnóstico riguroso como línea de base para medir cada cambio e impacto posterior, segundo la participación comunitaria como una condición sine qua non para que este proceso sea exitoso y efectivo y finalmente el ajuste al POT, como un documento guía donde se establecen las pautas y lineamientos del reasentamiento. 


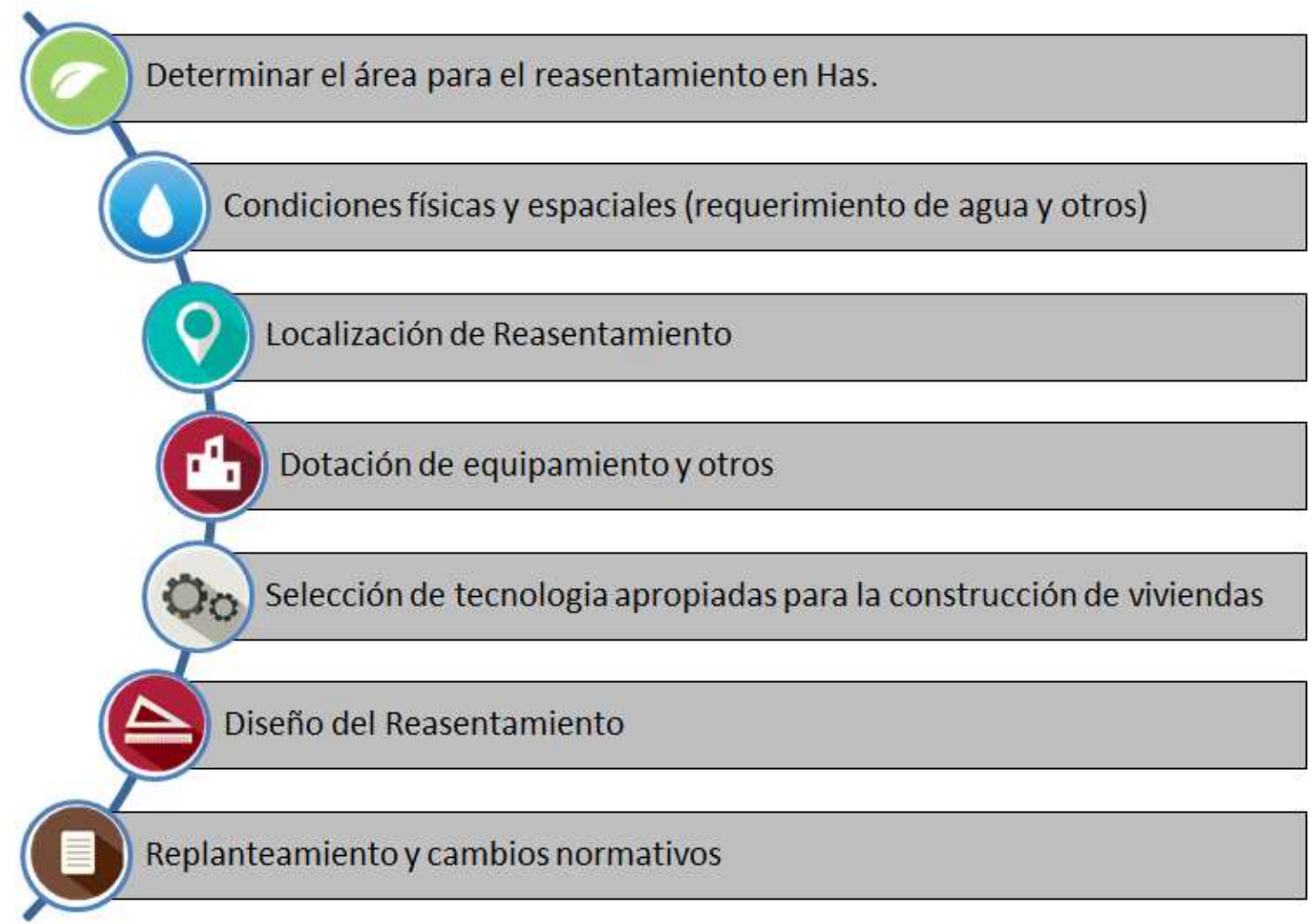

Figura 9. Proceso de planificación de Reasentamiento

Fuente: Esta Investigación

\section{BIBLIOGRAFÍA}

AGUDELO Suárez, Omar y Carlos (2011) Gestión del riesgo y manejo de crisis por desastres en instalaciones hospitalarias en el municipio de Tumaco, Universidad del Valle

ARDILA, Martha, (2012) El pacifico latinoamericano y su inserción internacional. Universidad Javeriana

BAERISWY, Sergio (2014) PRBC 18

BANCO MUNDIAL COLOMBIA, (2012) Análisis de la gestión del riesgo de desastres en Colombia, un aporte para la construcción de políticas publicas

Eco aldea para el consejo comunitario de Chanzara, Santa Barbara Iscuande Nariño, (2014), Universidad Piloto de Colombia

CARDONA A. Omar Darío (2005) Indicadores de riesgo de desastre y gestión de riesgos: programa para América Latina y el Caribe.

CARTES, Iván (2014) Premio Nacional de Urbanismo. Gobierno de Chile

CORREA, helena, otros. (2011) Reasentamiento preventivo de poblaciones en riesgo de desastres. Experiencias en Latinoamérica. The World Bank, 224.

GUERRERO Derly Sofia, (2011) Construcciones palafítica, recomendaciones de política pública en el litoral pacífico. Procuraduría GN, 2011. 
DIMAR Dirección General Marítima. Estudio y levantamiento predio propiedad de la alcaldía del municipio de San Andrés de Tumaco, en el cual se pretende la reubicación de 1000 familias asentadas dentro del casco urbano y que se encuentran en zonas de alto riesgo. 2008

FERNANDEZ, (2013) Hábitat vulnerable en situación de emergencia por desastres Naturales, Bio Bio Chile

INGEOMINAS. Mapa de zonificación geotécnica por licuación del área urbana del municipio de Tumaco y sus zonas aledañas. 2003

MOSQUERA y GNISET Gilma, Jacques Aprile, (1999) Urbanismo y Vivienda en las Ciudades del Pacífico: el Caso de Tumaco. Universidad del Valle. Citce.

MOSQUERA Gilma (2011) Vivienda y arquitectura tradicional en el pacifico colombiano.

MOSQUERA, Gilma, (1999) Jacquesm Aprile, Habitats y habitantes del pacifico. Universidad del Valle,

POT. Plan de Ordenamiento Territorial del Municipio de Tumaco. 2008-2019. (POT Tumaco, 2008).

RAMIREZ, Fernando, o. (2012) Análisis de la gestión del riesgo de desastres en Colombia, un aporte para la construcción de políticas públicas, Banco Mundial Colombia, 436p.

SALDARRIAGA, Alberto (2002) La arquitectura como experiencia, Espacio, cuerpo y sensibilidad

VARELA Barrios, Edgar, (2012) Política pública portuaria, un reto para el desarrollo competitivo del pacifico. U san Buenaventura. 